\title{
Endostar Rebuilding Vascular Homeostasis and Enhancing Chemotherapy Efficacy in Cervical Cancer Treatment
}

This article was published in the following Dove Press journal: OncoTargets and Therapy

\section{Liming Guan}

Department of Obstetrics and Jing'an District, Shanghai, 200070, People's Republic of China

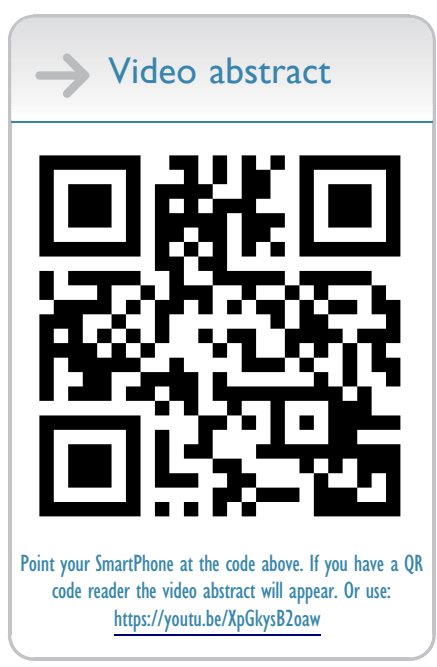
Gynaecology, Zhabei Central Hospital,

Background: The incidence rate of cervical cancer is the highest in the reproductive tract and is not sensitive to chemotherapy. An appropriate amount of anti-angiogenic agents can reconstruct tumor blood vessels in a short period of time and form vascular homeostasis, increase the function of blood vessel perfusion and reverse the multidrug resistance of chemotherapy, which is also called "vascular normalization." Endostar (a recombinant human endostatin) was developed by China and as a multi-target anti-angiogenesis agent. Many reports about endostar involved the treatment of non-small cell lung cancer, fewer reports are on cervical cancer.

Purpose: To determine whether endostar can rebuild tumor vascular homeostasis and enhance chemotherapy effects for patients with cervical cancer.

Methods: In this study, the patients with cervical cancer within stage IIB2 were selected, endostar combined with cisplatin+paclitaxel neoadjuvant chemotherapy (NACT) before radical surgical operation was adopted, patients outcome and adverse reaction were followed up. The changes of tumor vascular structure and perfusion function before and after endostar given were evaluated by histopathology and dynamic contrast-enhanced magnetic resonance imaging (DEC-MRI). VEGFNotch signal pathway was detected for the regulating mechanism of vascular proliferation in different groups. GraphPad Prism 6 software was used for statistical analysis of the study results.

Results: Endostar enhanced the short-term (2 year) overall survival (OS), progression-free survival (PFS) rates for cervical cancer patients. All the same, endostar increased long-term (5 year) OS for cervical cancer patients. Endostar therapy exhibited with mild adverse reaction. MRI showed endostar+NACT further reduce tumor volume than NACT alone. The parameters of Ktrans, Ve for DEC-MRI in endostar group exhibited obviously increase than NACT group. Tumor vascular maturation index $\alpha$-SMA/CD31 in endostar group increased obviously than NACT group, correspondingly Ki67 staining for tumor proliferative rates, lymphovascular space invasion in endostar group further declined than NACT group. The genes and proteins expression of VEGFR2, Notch1, Notch4, Dll4, Jag1 were obviously downregulated in endostar group comparing to NACT group.

Conclusion: Endostar restored vascular homeostasis in cervical cancer temporarily, enhanced chemotherapeutic agents effects in cervical cancer, increased patient OS ratio. Endostar+NACT treatment may provide a new target therapy for cervical cancer.

Keywords: vascular homeostasis, cervical cancer, chemotherapeutic resistance

\section{Background}

Department of Obstetrics and

Gynaecology, Zhabei Central Hospital,

Jing'an District, Shanghai 200070 People's

Republic of China

Email guanlimingI@I26.com
Uterine cervical cancer is the second most common cancer and one of the leading causes of cancer death for women, after breast cancer. Radiation therapy is regarded as a standard treatment just like surgery, but radiation can induce radiation colitis, 
loss of ovarian function and sexual capacity, these shortcomings make it only as an alternate option. Neoadjuvant chemotherapy (NACT) is used to treat local advanced cervical cancer in Italy, Japan and Korea. Application of NACT before surgery or before radiotherapy can reduce tumor load, tumor cell micrometastasis and lymph nodes invasion. But NACT is not more than $87.5 \%$ effective against cervical cancer. Ineffective NACT can lead to tumor progression, increase the difficulty of surgery, and affect treatment outcome of patients. ${ }^{1,2}$

Recombinant human endostatin (endostar) independently developed in China could inhibit the expression of VEGF, which played an anti-angiogenic role in multiple targets and has no drug resistance. It was mainly applied in the treatment of non-small cell lung cancer, and few reports related to the treatment of cervical cancer.

The main mechanism of endostar is to block the formation of tumor neovascularization by inhibiting the migration of vascular endothelial cells. As an antiangiogenic agent, endostar can improve the abnormal tumor structure, increase vascular perfusion within the validity of the agent. This process is called tumor vascular normalization. Such normalized tumor blood vessels can transport more chemotherapeutic agents to tumor tissues, make cytotoxic agents more effectively. ${ }^{3}$

In order to improve the efficacy of chemotherapy, endostar can be used to improve NACT reactivity, DECMRI can be used to monitor NACT reactivity in real time. DCE-MRI was based on T1-weighted images and then injected with contrast agent to obtain a series of images of contrast agent transport, distribution and outflow of tumor tissue. These images can be used to analyze the dynamic information of tumor vascular permeability and blood perfusion. DCE-MRI can monitor the dynamic distribution of tumor vascular perfusion before and after drug treatment. DCE-MRI can acquire the data about tumor blood vessel penetrability and perfusion. Pharmacokinetic indexes can obtain tumor blood vessel variation. It is reported that DCE-MRI indexes can predict tumor response to treatment and be used as a reliable method to follow up the changes of tumor blood vessels. ${ }^{4}$

We adopted endostar combined with cisplatin+paclitaxel (PT) NACT in cervical cancer patients before surgical operation, the first aim in this study was to follow up the patients prognosis; the second aim was to determine vascular hemodynamic change by DEC-MRI before and after endostar given; the third aim was to validate vascular maturation index via $\alpha$-SMA/CD31 staining and tumor proliferative change via Ki67 staining in cervical patients before and after endostar+NACT therapy. The regulating mechanism of VEGF-Notch connection in this course was explored.

\section{Methods}

\section{Patient Features}

From March 2014 to August 2014, 145 women with cervical cancer were enrolled to Zhabei Central Hospital, among whom 138 patients were included in this study. One hundred thirty eight patients gave informed consent and were fully evaluated for eligibility to participate in the study. The inclusion and exclusion criteria were met by 138/145 (95.2\%) patients, and $7(4.8 \%)$ patients exited the study without treatment. Reasons for ineligibility included 4 patients rejected treatment in Zhabei Central Hospital; two patients with cervical cancer suffered lung function insufficiency and one with heart disease unable to withstand anesthesia were not qualified for the operation. These 138 patients received NACT + surgery within 6 months, next their prognoses were followed up from September 2014 to September 2019. These patients come from within Shanghai, the Yangtze River Delta, and from all over China.

The number of subjects required was calculated via PASS Sample Size Software.

\section{The Inclusion Criteria}

The diagnosis of cervical cancer was based on cervical biopsy detection; no distant metastasis by auxiliary examination; stage within IIB2 according to FIGO 2018 classification cancer of the cervix uteri, i.e., the tumor invades beyond the uterus, but has not extended onto the lower third of the vagina or to the pelvic wall. ${ }^{5}$ Vascular or lymphatic invasion does not change the staging, we would follow up vascular or lymphatic involvement via pathologic changes.

Existence of cervical cancer foci that were measurable via response evaluation criteria in solid tumors (RECIST) standards; first diagnosis of cervical cancer, without previous radiotherapy or chemotherapy. Patients with cervical cancer were without coagulation disorders and myocardial disease, diabetes mellitus not controlled, or other complicated diseases. No history of drug or food allergy. Karnofsky scores $>70$; estimated OS $>6$ months. No serious complications hindering chemotherapy.

\section{Exclusion Criteria}

Distant metastasis; Karnofsky (KPS) score $<70$; combined with heart, kidney failure, and any other conditions 
contraindicated for chemotherapy such as infection, myocardial infarction, unstable angina pectoris, congestive heart failure, or uncontrolled arrhythmias, immunosuppressive therapy; pregnancy or lactation; severe bone marrow dysfunction; bleeding tendency; poor compliance, or cognitive disorders; drug abuse or alcohol addiction.

The average age of the 138 patients was 48 years, ranging from 32 to 65 years. According to FIGO standard of cervical cancer, the clinical diagnosis stage of the patients in this study is from stage IB2 to stage IIB2. The pathological types of patients were mainly squamous carcinoma, and a few were adenocarcinoma. The ages, FIGO staging, pathological types, tumor differentiations and tumor maximum diameters were compared between two groups at the beginning of treatment. There was no significant different for patient features between the 2 groups. The general features of patients see Table 1 .

This study was approved by the Ethics committee of Zhabei Central Hospital (No.2015-35). All patients in this study were thoroughly informed of the benefits, curative effects, potential risks of the condition and management alternatives, treatment costs and uncertainties related to cervical cancer radical operation, or a NACT protocol treatment followed by surgery. All patients gave written consent to participate in the study. There were no conflicts of interest.

After appropriate counseling, patients were referred to our cancer treatment center. Both patients and doctors were blinded to group allocation. Enrolled patients were then sequentially numbered. Patients labeled with odd numbers were given NACT+surgical operation (NACT group), while patients labeled with even numbers received endostar+NACT followed by surgery (endostar group). The extent of surgery after NACT remains the same, i.e., radical hysterectomy and pelvic lymphadenectomy. The ratio of two groups was 1:1 for the 138 cases of patients, Figure 1 CONSORT flow diagram.

\section{Endostar-Targeted Therapy and Chemotherapy}

Endostar (Medizin Biopharmaceutical Co., Ltd. China) was continuously given intravenously at a dose of $15 \mathrm{mg} /$ $\mathrm{d}$ from day 1 to day 14, the next 7 days as an interval.

For NACT, paclitaxel were administered intravenously at a dose of $175 \mathrm{mg} / \mathrm{m}^{2}$ on day 1 . Cisplatin was given in a vein at a dose of $70 \mathrm{mg} / \mathrm{m}^{2}$ on day 1 , the next 3 weeks were as an interval.

The patients in the endostar group received 2 courses of endostar+NACT treatment, while patients in NACT group accepted 2 courses of NACT alone.

For antiemetic therapy, ondansetron was given intravenously at dose of $8 \mathrm{mg} 15$ minutes before chemotherapy, 4 hours after chemotherapy and 8 hours after chemotherapy, and every $8 \sim 12$ hours after the cessation of chemotherapy.

To prevent allergy reaction, dexamethasone was administrated orally at dose $20 \mathrm{mg} 12$ hours and 6 hours respectively before chemotherapy.

Hydration was performed during chemotherapy period.

\section{Recent Efficacy Evaluation}

Before and after NACT treatment, physical examination, DCE-MRI detection and cervical cancer biopsy were carried out. After radical operation of cervical cancer, the specimens were removed for routine and immunohistochemical pathological examination.

Table I The General Features of Patients

\begin{tabular}{|c|c|c|c|c|c|}
\hline \multicolumn{2}{|c|}{ Parameters $(\mathrm{n} I=\mathrm{n} 2=69)$} & Endostar Group & NACT Group & $X^{2}$ or t Value & $P$ value \\
\hline \multicolumn{2}{|l|}{ Age(Years) } & $48 \pm 9$ & $51 \pm 12$ & $\mathrm{t}=1.386$ & 0.1691 \\
\hline FIGO staging & $\begin{array}{l}\text { IB } \\
\text { IIA+IIB }\end{array}$ & $\begin{array}{l}32 \\
37\end{array}$ & $\begin{array}{l}33 \\
36\end{array}$ & 0.02908 & 0.8646 \\
\hline Pathological types & $\begin{array}{l}\text { Squamous carcinoma } \\
\text { Adenocarcinoma }\end{array}$ & $\begin{array}{l}60 \\
9\end{array}$ & $\begin{array}{l}63 \\
6\end{array}$ & 0.6732 & 0.4119 \\
\hline Tumor differentiation & $\begin{array}{l}\text { Poorly } \\
\text { Moderately } \\
\text { Highly }\end{array}$ & $\begin{array}{l}17 \\
36 \\
16\end{array}$ & $\begin{array}{l}15 \\
38 \\
16\end{array}$ & 0.1791 & 0.9144 \\
\hline Tumor diameter & $\begin{array}{l}<4 \mathrm{~cm} \\
\geq 4 \mathrm{~cm}\end{array}$ & $\begin{array}{l}39 \\
30\end{array}$ & $\begin{array}{l}41 \\
28\end{array}$ & 0.1190 & 0.7302 \\
\hline
\end{tabular}




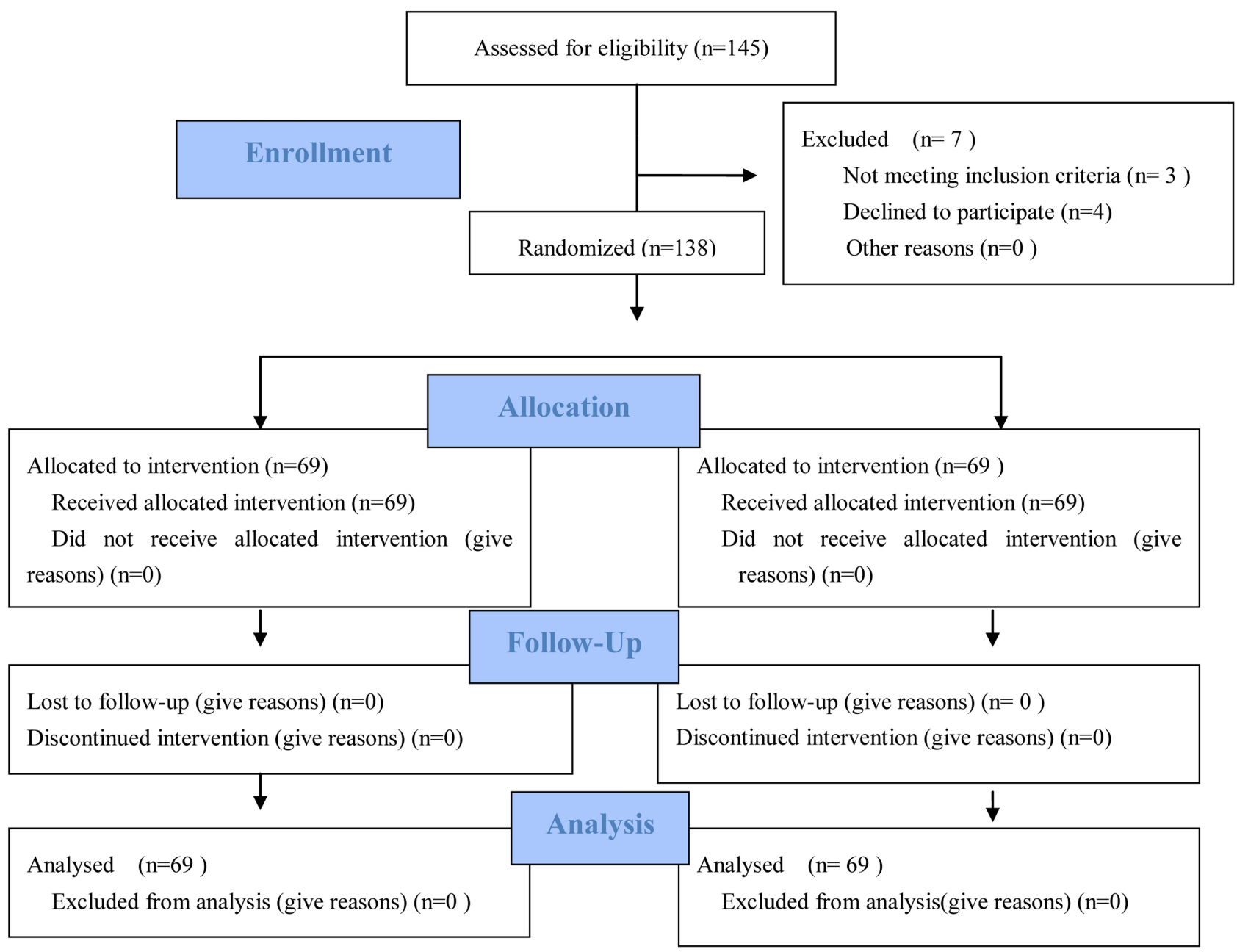

Figure I CONSORT flow diagram. Adapted from Schulz KF, Altman DG, Moher D, for the CONSORT Group (2010) CONSORT 2010 Statement: Updated Guidelines for Reporting Parallel Group Randomised Trials. PLoS Med 7(3): el 00025 I. Copyright: @ 2010 Schulz et al. Creative Commons Attribution License. ${ }^{6}$

Three months after surgery operation, the recent efficacy of treatment was evaluated according to physical examination, gynecological examination, liquid-based cytology, pelvic ultrasound, squamous cell antigen detection.

Quality of life assessment was referred to KPS changes, decline: KPS score decreases $\geq 10$ points; Stable: no significant change in KPS score; Improvement: KPS score increased $\geq 10$ points and maintained over 4W. Effectiveness $=$ improvement + stability.

Drug toxicity: grade 0-iv were divided according to international standard for toxicity of cancer (NCICTC), WHO standards for acute, subacute toxic and side effects of anticancer drugs. Adverse events were included: blood routine, liver and kidney function, gastrointestinal reactions and peripheral neurotoxicity and cardiotoxicity weekly.

According to RECIST criteria, the short-term efficacy was divided into complete response (CR), partial response $(\mathrm{PR})$, stable disease (SD), and progressive disease (PD).
The response rate (RR) was summed up by the rates of CR and $P R$, i.e., response rate $(R R)=C R+P R$. Disease control rate $(\mathrm{DCR})=\mathrm{CR}+\mathrm{PR}+\mathrm{SD}$.

\section{MRI Protocol}

MR images were acquired on 3.0T scanner (Siemens magnetom verio, Germany) from eight-channel surface phased-array coil.

Sagittal imaging thick 5-mm, T2-weighted images (echo time, $\mathrm{TE})=80$ milliseconds; repetition time $(\mathrm{TR})=4500$ milliseconds; two excitations, Both axial T1, T2-weighted images thick were 5-mm. Exciting number was 2. Pre-contrast images were acquired, next a dose of $0.22 \mathrm{mmol} / \mathrm{kg}$ iohexol was given via a pressure injector at $2 \mathrm{~mL} / \mathrm{s}$, at last a $20-\mathrm{mL}$ saline solution was flushed.

It took about 35 minutes to achieve this whole MRI detection. The image data were input into MATLAB software for analysis. The parameters of MR images included volume 
transfer constant (Ktrans), extravascular extracellular volume fraction (Ve), $\operatorname{kep}(=\mathrm{Ktrans} / \mathrm{ve}$, the efflux constant). The value of above parameters responsive to cervical cancer were evaluated via receiver operating characteristic curve (ROC).

\section{Tumor Volume Count}

Tumor volume and the maximum diameter of tumor were evaluated quantitatively on the T2-weighted MR images. Tumor volume is based as following formula:

Tumor volume $=$ tumor section number $\times$ tumor section thickness $\times$ tumor area

The slice numbers of tumor MR transversal images and the section thickness are summed up. Tumor area was measured the region within the outline of every tumor slice on axial T2weighted images. The longest tumor diameter basing on MR transversal images is considered as tumor diameter. ${ }^{7}$

\section{Long-Term Follow Up}

After surgery, the patients were followed up every 3 months over the first 2 years, every 6 months for the next 2 years; then subsequently once a year. The patient performed physical and gynecological examination, pelvic ultrasound and MRI in order to find out tumor local relapse and distant metastasis in time. Because overall survival (OS) rates were monitored, the patients were followed for at least 5 years.

The parameters for analysis included: OS, the period from the first day of diagnosis to death. Progression-free survival (PFS), local relapse-free survival (LRFS), distant metastasis-free survival (DMFS).

\section{Pathological Evaluation}

The pathological indexes included deep stromal invasion, excision margin status, vaginal invasion, parametrial involvement, lymphovascular space involvement, lymph node metastasis.

Histological response refer to the criteria of tumor regression grade defined by the Japan Society for Cancer Therapy. When less than two-thirds of the cancer cells undergo degeneration, necrosis, liquefaction, they are defined as histological nonresponders. When more than two-thirds of the cancer cells degenerate, liquefy, even if the all tumor is replaced by fibrosis tissue, they are defined as histological responders. ${ }^{8}$

\section{Vascular Maturation and Cell Proliferation Detection}

Cervical cancer tissues from the endostar group and the NACT group were fixed in $10 \%$ formalin, embedded in paraffin, sectioned, and placed on slides. The slides were blocked with blocking buffer and detected by primary Abs specific for CD31, Ki67, and $\alpha$-SMA following the manufacturer's instructions.

Then sections were detected by the secondary Abs and labeled by $3,3^{\prime}$-diaminobenzidine.

The following indices were quantified: microvessel density (MVD), tumor cell proliferation, endothelial cell coverage, and nuclear density.

MVD count: isolated endothelial cells and cell clusters that were positively stained with CD31 positive with or without visible lumina were counted as separate microvessels. MVD was examined at $\times 400$ magnification under a light microscope. The five highest MVD counts per tumor region of interest were averaged and used for the statistical analysis.

Utilizing the same region of interest, coverage of $\alpha$ SMA stained endothelial cells was calculated. The vessel maturation index (VMI) was defined as the ratio of $\alpha$-SMA-stained vasculature to CD31-stained vasculature.

Tumor cell proliferating ratio was defined by the percentage of Ki67 positive stained nuclei per total nuclei within the viable tissue regions of the tumor.

The details are provided in reference. ${ }^{9}$

\section{VEGF-Notch Signal Pathway Detection}

Cervical cancer vascular endothelial cells were isolated by tissue block combined with trypsin digestive method. The genes and proteins expression involving VEGF-Notch signaling pathway were analyzed.

\section{Real-Time PCR Detection}

Total RNAs were isolated from cultured cells by a total cellular RNA isolation kit (Beijing Solaibao Technology Co. Ltd.). Then, the RNAs from the samples were reverse transcribed using a Takara reverse transcription kit. Realtime quantitative PCR was performed by an R\&D Biosystems real-time PCR instrument. The cDNA samples were labelled by SYBR Green. The markers included VEGF, VEGFR2, Notch1, Notch4, Dll4, JAG1. According to temperature differences, PCR detection mainly included three steps: degeneration, annealing, and extension. The primer sequences are listed in Table 2. The process was performed for 30 cycles according to the manufacturer's instructions. Gene expression levels were evaluated using the $2^{-\Delta \Delta \mathrm{Ct}}$ method. $\beta$-actin was used as an internal reference. 


\section{Western Blot Analysis}

Proteins were extracted from the cells via an NP-40 lysis buffer containing a protease inhibitor formula. The total protein was quantified by bicinchoninic acid (BCA) method. Afterwards it was mixed with a reductive agent, an equal amount of protein (70ug) were separated via sodium dodecyl sulfate polyacrylamide gel electrophoresis (SDS-PAGE) and transferred to polyvinylidene difluoride (PVDF) membrane for blotting analysis. Membranes were blocked by bovine serum albumin (BSA) overnight and conjugated with primary antibodies VEGF, VEGFR, DLL-4, Jag1, Notch-1, 4; and incubated with secondary antibody connected with horseradish peroxidase. The membranes were labeled by 3,3'diaminobenzidine. $\beta$-actin band was acted as an internal reference. After staining and exposure, the protein bands in the film were analyzed via Image J software.

\section{Statistic Analysis}

In this study, GraphPad Prism 6 was used for statistical analysis. Kaplan-Meier method was used to evaluate the patients prognosis. The quantitative data were presented as mean \pm SD. The difference of two groups was compared with Student's $t$-tests. When the groups were over two groups, two-way ANOVA was adopted. Categorical variables were expressed as percentages (\%), and evaluated

Table 2 The Primer Sequences of VEGF-Notch Pathway

\begin{tabular}{|c|c|c|}
\hline Name & The Sequence of Primer $\left(5^{\prime}-3^{\prime}\right)$ & Product (bp) \\
\hline VEGF & $\begin{array}{l}\text { F: TGCCCACTGAGGAGTCCAAC } \\
\text { R: TGGTTCCCGAAACGCTGAG }\end{array}$ & 336 \\
\hline VEGFR2 & $\begin{array}{l}\text { F: AAGAGATTTGTTCCGGATGG } \\
\text { R:CGGCAGATAGCTCAATTTCA }\end{array}$ & 360 \\
\hline Notch I & $\begin{array}{l}\text { F: GGAAGTTGAACGAGCATAGTCC } \\
\text { R: GCATGATGCCTACATTTCAAGA }\end{array}$ & 430 \\
\hline Notch4 & $\begin{array}{l}\text { F:GCTCCTTCAACTGCCTCTGTCC } \\
\text { R:GCACACTCGTTGGTCTCCACCT }\end{array}$ & 430 \\
\hline DII4 & $\begin{array}{l}\text { F: ATTGCCAACAGCCTATCT } \\
\text { R: CCATCCTCCTGGTCCTTA }\end{array}$ & 388 \\
\hline Jagl & $\begin{array}{l}\text { F:CTGTGGCTTGGATCTGTTGCT } \\
\text { R:CGTTGTTGGTGGTGTTGTCCT }\end{array}$ & 505 \\
\hline$\beta$-actin & $\begin{array}{l}\text { F: ATCATGTTTGAGACCTTCAACA } \\
\text { R: CATCTCTTGCTCGAAGTCCA }\end{array}$ & 318 \\
\hline
\end{tabular}

Abbreviations: $F$, forward; $R$, reverse. with Fisher's exact test or Chi-square analysis. $\mathrm{P}<0.05$ was considered statistically significant.

\section{Results}

\section{Endostar Enhanced NACT Short-Term Treatment Effects}

In this study, the short-term (2 years) efficacy of endostar + NACT was evaluated, CR, PR and SD rates in the endostar group were $79.7,10.1 \%$ and $5.8 \%$, respectively. While CR, $\mathrm{PR}$ and SD rates in NACT alone group were $52.1 \%, 20.2 \%$ and $4.3 \%(3 / 69)$, separately. So chemotherapeutic response rates (RR) in endostar group and NACT group were 89.9\% vs.. $72.4 \%$ respectively, there was obviously different between the 2 groups (Fisher's exact test; $\alpha<0.05$, $\mathrm{P}=0.0155$ ). The disease control rates (DCR) in endostar group and NACT group were $95.6 \%$ vs. $76.8 \%$, respectively. There was obviously different between the 2 groups (Fisher's exact test, $\alpha<0.05, \mathrm{P}=0.0001$ ). This results indicated endostar increased chemotherapeutic agents effects in short-term efficacy compared with NACT alone, see Table 3.

\section{Endostar Enhanced NACT Long-Term Efficacy}

The patients were followed up from 38 to 57 months for longterm efficacy, the average time was 49.6 months (region: $38.3-65.3$ months), OS were $87.0 \%$ vs. $71.0 \%$ in endostar and NACT group, there was obviously different between 2 groups (Fisher's exact test, $\alpha<0.05, \mathrm{P}=0.0087$ ). These included 9 patients who died in the endostar group and 20 patients who died in the NACT group.

The 2-year PFS rates in the endostar group and the NACT group were $97.1 \%$ and $84.1 \%$, respectively. There was significant differences between the two groups (Fisher's exact test, $\mathrm{P}=0.0014, \alpha<0.05$ ). These included 2 cases tumor progression in endostar group and 11 cases in NACT group.

The 2-year LRFS in the two groups were $95.7 \%$ and $85.5 \%$ separately (Fisher's exact test, $\alpha<0.05, \mathrm{P}=0.0329$ ). There was statistically significant between two groups. These included 3 patients local relapse in the endostar group and 10 patients local relapse in the NACT group.

The 2-year distance metastasis-free survival rates in the two groups were $93.3 \%$ and $81.4 \%$ separately (Fisher's exact test, $\alpha<0.05, P=0.0193$ ), there was statistically significant between two groups. These included 5 cases distance metastasis in endostar group and 13 cases in NACT group, see Table 3. 
Table 3 The Efficacy of Endostar+NACT for Cervical Patients

\begin{tabular}{|l|l|l|l|l|l|}
\hline \multicolumn{2}{|l|}{ Efficacy (nI=n2=69) } & Endostar Group & NACT Group & Fisher's Exact Test & P value \\
\hline Short-term efficacy(\%) & Complete response & $55(79.7 \%)$ & $36(52.1 \%)$ & $\alpha<0.05$ & 0.0002 \\
& Partial response & $7(10.1 \%)$ & $14(20.2 \%)$ & $\alpha>0.05$ & 0.1539 \\
& Stable & $4(5.8 \%)$ & $3(4.3 \%)$ & $\alpha>0.05$ & 1.0000 \\
& Response rate & $62(89.9 \%)$ & $50(72.4 \%)$ & $\alpha<0.05$ & 0.0155 \\
& Disease control & $66(95.6 \%)$ & $53(76.8 \%)$ & $\alpha<0.05$ & 0.0001 \\
\hline \multirow{2}{*}{ Long-term efficacy(\%) } & Overall survival & $60(87.0 \%)$ & $49(71.0 \%)$ & $\alpha<0.05$ & 0.0355 \\
& Progression-free survival & $67(97.1 \%)$ & $58(84.1 \%)$ & $\alpha<0.05$ & 0.0028 \\
& Local relapse-free & $66(95.7 \%)$ & $59(85.5 \%)$ & $\alpha<0.05$ & 0.0329 \\
& Metastasis-free survival & $64(93.3 \%)$ & $56(81.4 \%)$ & $\alpha<0.05$ & \\
\end{tabular}

The survival rates of patients are reported in Figure 2. The results indicated that endostar+NACT obviously enhances the long-term effects better than NACT alone.
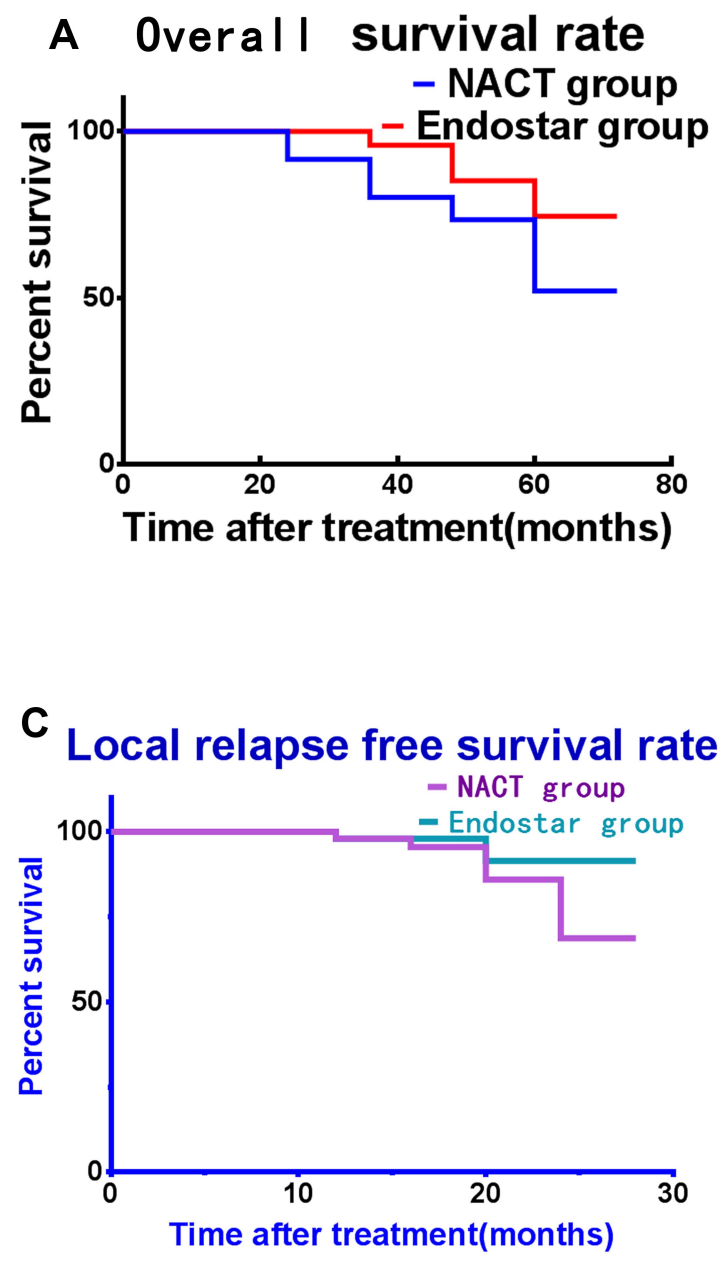

\section{Endostar Reduced NACT Toxicity}

To assess chemotherapy adverse responses, Common Terminology Criteria Adverse Events Version 4.0 from National Cancer Institute (NCI) was adopted. The

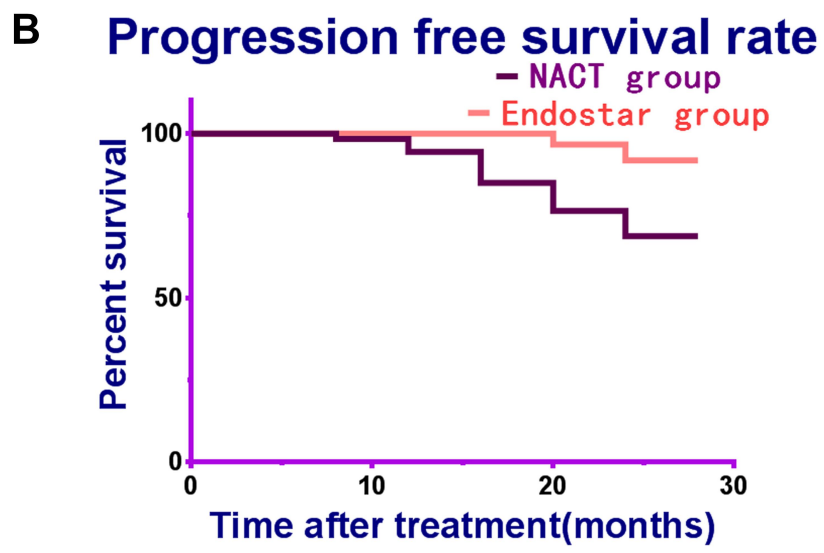

$\mathbf{D}$

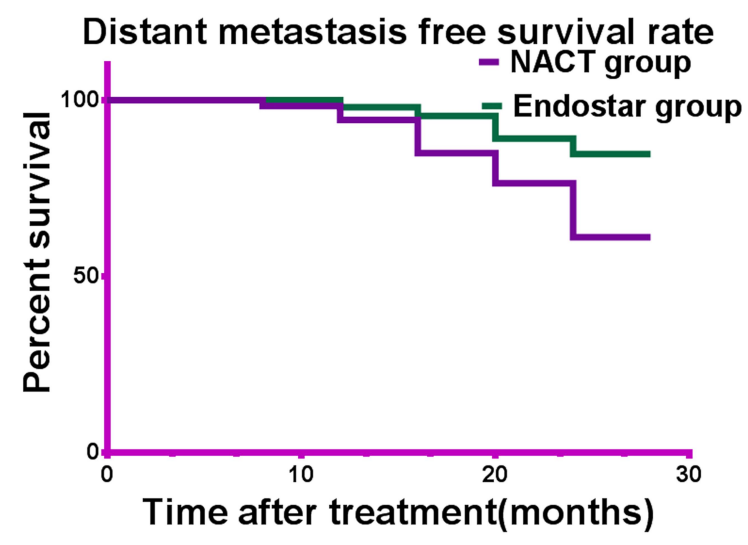

Figure 2 The prognosis for the patients present in curves. (A) The overall survival rate increased obviously in endostar group than NACT group. (B) The progression-free survival rate enhanced in endostar group. (C) The local relapse-free proportion increased in endostar group. (D) The distance metastasis-free survival rate enhanced significantly in endostar group than NACT group. 
general adverse effects included myelosuppression, hair loss, gastrointestinal reactions, weightlessness, see Table 4.

In the endostar group, the incidence of myelosuppression (such as leukocytopenia) in grades 0,1 and 2 were $55.0 \%$, $18.0 \%$, and $27.0 \%$, differently. In the NACT group, the incidence of myelosuppressionin in grades $0,1,2$, and 3 were $8 \%, 20.5 \%, 50.3 \%$, and $21.2 \%$, differently. There were statistical differences between the 2 groups (Chi-square $=63.16, \mathrm{P}<0.0001, \alpha<0.05$ ).

In the endostar group, the incidence of gastrointestinal reactions in grades 0,1 , and 2 were $38.0 \%, 49.0 \%$, and $13.0 \%$, separately. The incidence gastrointestinal reactions in grades 2, 3 and 4 were $75.4 \%, 17.5 \%$ and $7.1 \%$ in NACT group, differently. There were distinct differences between 2 groups (Chi-square=32.08, $\mathrm{P}<0.0001, \alpha<0.05$ ).

In the endostar group, the incidence of weight loss in grades 1 and 2 were $75.2 \%$ and $24.8 \%$ separately. In the NACT alone group, the incidence weight loss in grades 0 and 1 were $89.8 . \%, 10.2 \%$, separately. There were obvious differences between the 2 groups (Chi-square $=164.7, \mathrm{P}<$ $0.0001, \alpha<0.05)$.

In the endostar group, the incidence of oral ulcer in grades 0,1 were $78.0 \%, 22.0 \%$, separately. In NACT group, the incidence of oral ulcer in grades 0,1 , and 2 were $46.3 \%, 35.5 \%$ and $18.2 \%$, separately. The two groups had obvious differences (Chi-square $=29.22, \mathrm{P}<0.0001$, $\alpha<0.05$ ).

In the endostar group, the incidence of hair loss in grades 0,1 , and 2 were $45.0 \%, 49.0 \%$, and $6.0 \%$, separately. In the NACT group, the incidence of hair loss in grades 0,1 , and 2 were $23.5 \%, 56.4 \%$ and $21.1 \%$, separately. The two groups had obvious differences (Chisquare $=15.92, \mathrm{P}=0.0003, \alpha<0.05$ ).

In this study, the dysfunction of vital organs was not found in all patients, so endostar may partly relieve NACT adverse reactions.

\section{Endostar Enhanced NACT Tumor Volume Reduction Effects}

The change of tumor volume was monitored by MRI, it showed the tumor volume was $5.48 \pm 0.36 \mathrm{~cm}^{3}$ and 3.10 $\pm 0.32 \mathrm{~cm}^{3}(\mathrm{n} 1=69)$ before and after treatment in endostar group respectively. In contrast, the tumor volume was 5.39 $\pm 0.45 \mathrm{~cm}^{3}$ and $4.51 \pm 0.25 \mathrm{~cm}^{3}(\mathrm{n} 2=69)$ before and after treatment in NACT group. There was distinctly different between two groups after treatment (Unpaired $t$-test, $\mathrm{t}=14.20, \mathrm{P}<0.0001)$. The same result could be found from the maximum diameter basing on MRI measurement. So it exhibited that tumor volumes and maximum diameters further declined in endostar group than NACT group, see Figure $3 \mathrm{~A}$ and $\mathrm{B}$. As measured by tumor volume and maximum diameter changes, endostar enhanced the effects of NACT for cervical cancer patients.

\section{The Efficacy of Endostar Determined by DCE-MRI}

In this study, DCE-MRI was used to obtain cervical cancer perfusion and permeability status.

The ROC determines the optimal cutoff value for DCE-MRI detection. The critical point has the shortest distance from the top left corner to the ROC curve. On this point the tangent line of the ROC curve intersects with the ROC curve, the sum of sensitivity and specificity value is the highest at the same time with the least sum of false positives and false negatives value.

ROC analysis for DCE-MRI parameters were carried out between endostar and NACT group before and after treatment. Before treatment, ROC analysis showed area under the curve values of Ktrans, Ve, and kep (Ktrans/ ve) in endostar group were $0.35 \pm 0.02,0.56 \pm 0.03,0.59$ \pm 0.08 separately. These results were regarded as baseline parameters.

One week after treatment, the area under the curve values for Ktrans, Ve, kep were $0.54 \pm 0.03 \quad(\mathrm{t}=36.51$,

Table 4 Adverse Reaction of Endostar and NACT Groups

\begin{tabular}{|l|l|l|l|l|l|l|l|l|l|l|l|l|}
\hline \multirow{2}{*}{ Parameters(\%) } & \multicolumn{3}{l}{ Endostar Group } & \multicolumn{3}{l|}{ NACT Group } \\
\cline { 2 - 12 } & $\mathbf{0}$ & $\mathbf{I}$ & $\mathbf{2}$ & $\mathbf{3}$ & $\mathbf{4}$ & $\mathbf{0}$ & $\mathbf{I}$ & $\mathbf{2}$ & $\mathbf{3}$ & $\mathbf{4}$ & $\boldsymbol{X}^{\mathbf{2}}$ & $\mathbf{P}$ value \\
\hline Myelosuppression & 55 & 18 & 27 & 0 & 0 & 8 & 20.5 & 50.3 & 21.2 & 0 & 63.16 & $<0.0001$ \\
Gastrointestinal reactions & 38 & 49 & 13 & 0 & 0 & 0 & 75.4 & 17.5 & 7.1 & 0 & 28.82 & $<0.0001$ \\
Weight loss & 0 & 75.2 & 24.8 & 0 & 0 & 0 & 89.8 & 10.2 & 0 & 0 & 7.792 & 0.0052 \\
Oral ulcer & 78 & 22 & 0 & 0 & 0 & 46.3 & 35.5 & 18.2 & 0 & 0 & 29.22 & $<0.0001$ \\
Hair loss & 45 & 49 & 6 & 0 & 0 & 23.5 & 56.4 & 21.1 & 0 & 0 & 15.92 & 0.0003 \\
\hline
\end{tabular}



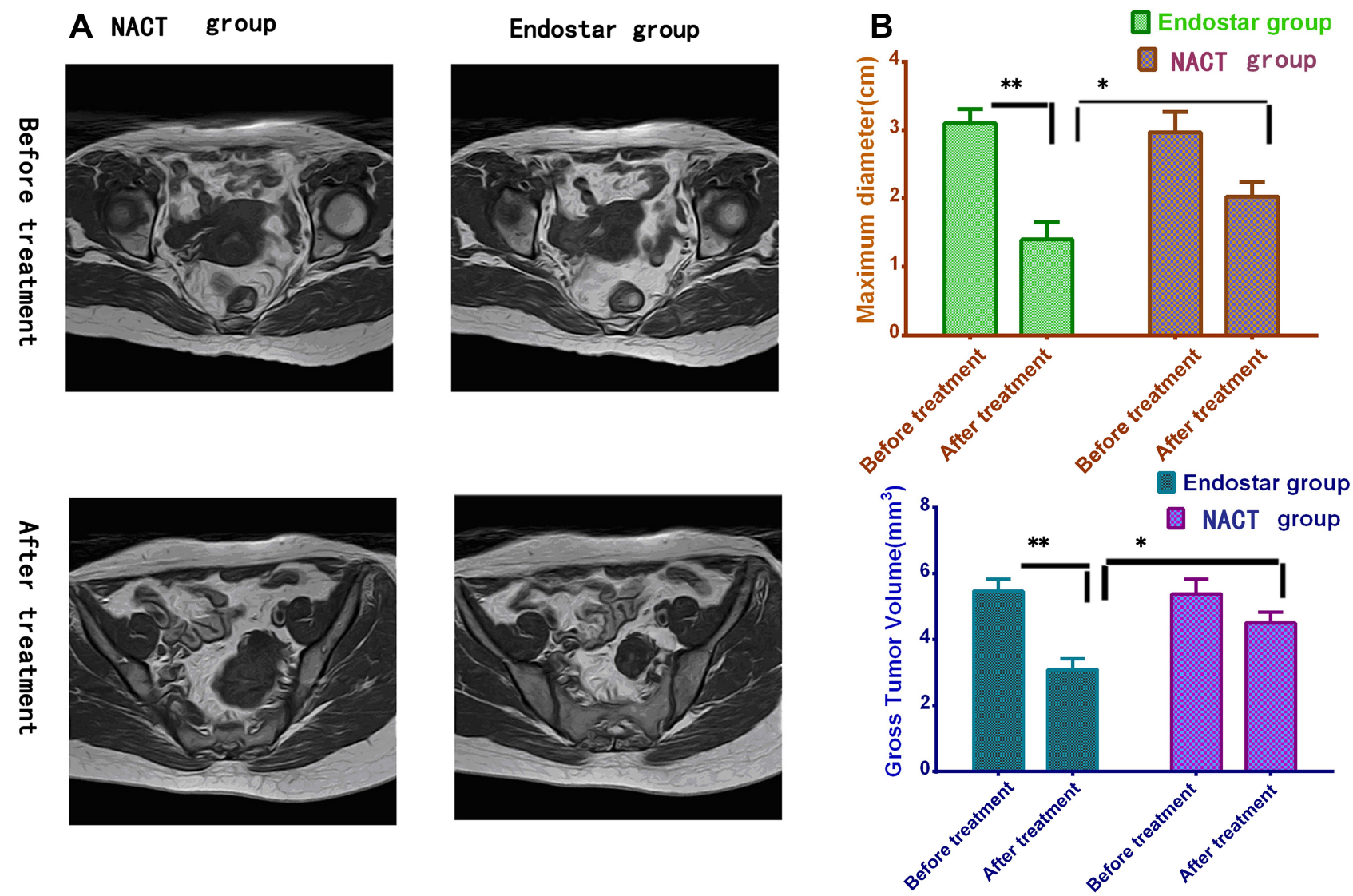

Figure 3 The sizes of cervical cancer measured by MR images. (A) The cervical cancer detected by MR images before and after treatment. (B) The tumor volumes and maximum diameters declined obviously in endostar than NACT group after treatment (Unpaired $t$-test, $* \mathrm{P}<0.05 ; * * \mathrm{P}<0.01$ ).

$\mathrm{P}<0.0001, \mathrm{n} 1=69), 0.82 \pm 0.04(\mathrm{t}=36.03, \mathrm{P}<0.0001), 0.62$ $\pm 0.05(\mathrm{t}=1.469, \mathrm{P}=0.1452)$ in endostar group, there were significant different before and after treatment for Ktrans and Ve value, see Figures 4A and 5A.

Before treatment, the values of Ktrans, Ve, and kep were $0.38 \pm 0.08,0.61 \pm 0.10,0.64 \pm 0.13$ in NACT group. The values of Ktrans, Ve, kep were $0.40 \pm 0.07(\mathrm{t}=1.563$, $\mathrm{P}=0.1204, \mathrm{n} 2=69), \quad 0.58 \pm 0.12 \quad(\mathrm{t}=1.595, \mathrm{P}=0.113), 0.67$ $\pm 0.15(\mathrm{t}=1.255, \mathrm{P}=0.2115)$ after treatment in NACT group. There were no significant differences before and after treatment, see Figures $4 \mathrm{~A}$ and $5 \mathrm{~A}$.

When the cutoff of $\mathrm{K}^{\text {trans }}$ value was $0.425(\mathrm{~mL} / \mathrm{min})$, the sensitivity, specificity were 81.25 (95\% CI: 54.35 95.95), 93.75 (95\% CI: 69.77-99.84) separately, see Figure 4B.

When the cutoff of Ve value was $0.525(\mathrm{~mL} / \mathrm{mL})$, the sensitivity, specificity were 87.5 (95\% CI: 61.65-98.45), 93.75 (95\% CI: 69.65-97.94) separately, see Figure 5B.

When the cutoff of kep value was $0.325(\mathrm{~mL} / \mathrm{min})$, the sensitivity, specificity were 53.85 (95\% CI: 25.13-80.78), 84.62 (95\% CI: 54.55-98.08) respectively.

\section{The Efficacy of Endostar Evaluated by Pathology}

The efficacy for endostar+NACT treatment of cervical cancer stage within IIB was determined by tumor tissue pathologic changes. All the slides of excised specimens were reviewed and divided according to indexes mentioned above. The cervical deep stromal invasion in the endostar and NACT group was 33.3\% and 39.6\% separately. There was no obvious difference between two groups (Fisher's exact test, $\alpha>0.05, \quad \mathrm{P}=0.3782$ ). Similarly, there were not statistically different between two groups in the parameters of excision margin status, vaginal invasion, parametrial involvement (Fisher's exact test, $\alpha>0.05$ ), see Table 5 . The rates of tumor regression over two-thirds in the endostar group and the NACT group were $37.5 \%$ and $20.8 \%$ respectively, there was significantly different between two groups (Fisher's exact test, $\alpha<0.05, \mathrm{P}=0.0128$ ). Similarly, there were statistical differences between two groups in the detection of lymphovascular space involvement, lymph 

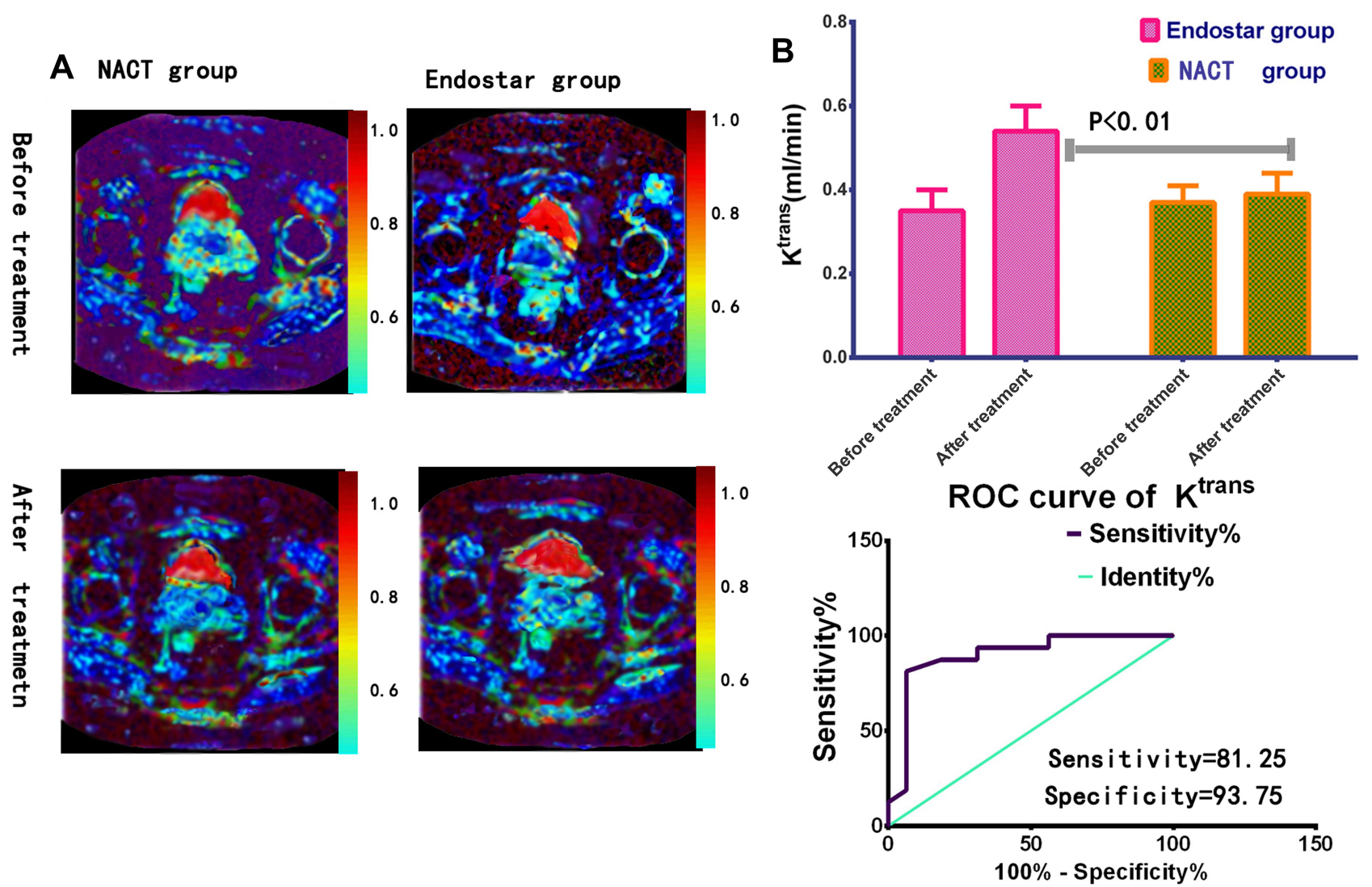

Figure 4 The Ktrans value detection in endostar treatment. (A) Parametric maps in different groups of volume transfer constant (Ktrans) were presented by DEC-MRI. (B) One week after treatment, Ktrans values of tumors increased distinctly in endostar than NACT group. Receiver operating characteristic (ROC) analysis between endostar and NACT group was carried out. The area under curve $(A \cup C)$ was $0.8965(\mathrm{P}<0.0 \mathrm{I})$.

node metastasis (Fisher's exact test, $\alpha<0.05$ ), see Table 5 .

\section{Vascular Maturation and Cell Proliferation}

Before the treatment, CD31 staining rates were $85.54 \pm 6.32$ vs $83.36 \pm 7.63$ in endostar group and NACT group respectively, there was no significant difference between the two groups (Unpaired $t$-test, $\mathrm{t}=1.503, \mathrm{P}=0.1362, \mathrm{n} 1=\mathrm{n} 2=69$ ). One week after treatment, CD31 staining rates in endostar group and NACT group were $45.43 \pm 4.35$ vs. $78.24 \pm 6.54$, respectively. This confirmed that MVD in the endostar group was relatively reduced more than that of the NACT group. There was significant difference between two group (Unpaired $t$-test, $\mathrm{t}=34.70, \quad \mathrm{P}<0.0001$, $\mathrm{n} 1=\mathrm{n} 2=69)$.

$\alpha$-SMA staining were $13.38 \pm 4.46$ vs $15.45 \pm 5.37$ before treatment. Seven days after treatment, $\alpha$-SMA label were $37.35 \pm 5.34$ vs $13.67 \pm 4.74$ in endostar group and NACT group. There was dramatic differences between the two groups (Unpaired $t$-test, $\mathrm{t}=27.90, \mathrm{P}<0.0001, \mathrm{n} 1=\mathrm{n} 2=69$ ). Before treatment, tumor pericyte density was at the same level, after treatment, pericyte rates obviously increased in endostar group.

VMI (the ratio of $\alpha$-SMA-stained vasculature to CD31stained vasculature) in the endostar group was $0.15 \pm 0.04$ and $0.28 \pm 0.05$ before and after treatment, separately. VMI was $0.18 \pm 0.05$ and $0.19 \pm 0.06$ in NACT group before and after treatment. There were significant differences between two groups after treatment (Unpaired $t$-test, $\mathrm{t}=214.3$, $\mathrm{P}<0.0001, \mathrm{n} 1=\mathrm{n} 2=69$ ). So endostar made the tumor vascular structure more mature than NACT group.

Before the treatment, Ki67 positive staining exhibited the same proliferative level in endostar group and NACT group $(42.5 \pm 3.3$ vs $43.6 \pm 5.4, \mathrm{n} 1=\mathrm{n} 2=69)$, there was no significant difference between the two groups (Unpaired $t$-test, $\mathrm{P}=0.2315, \mathrm{t}=1.204$ ). Seven days after treatment, Ki67 positive staining rates in endostar group and NACT group were $23.32 \pm 4.25 \%$ vs. $35.36 \pm 4.67 \%$, respectively, there were significant differences between two groups (Unpaired $t$-test, $\mathrm{t}=15.84, \mathrm{P}<0.0001$ ). This proved the tumor proliferative rates in the endostar group were reduced more than the NACT group (see Figure 6). 


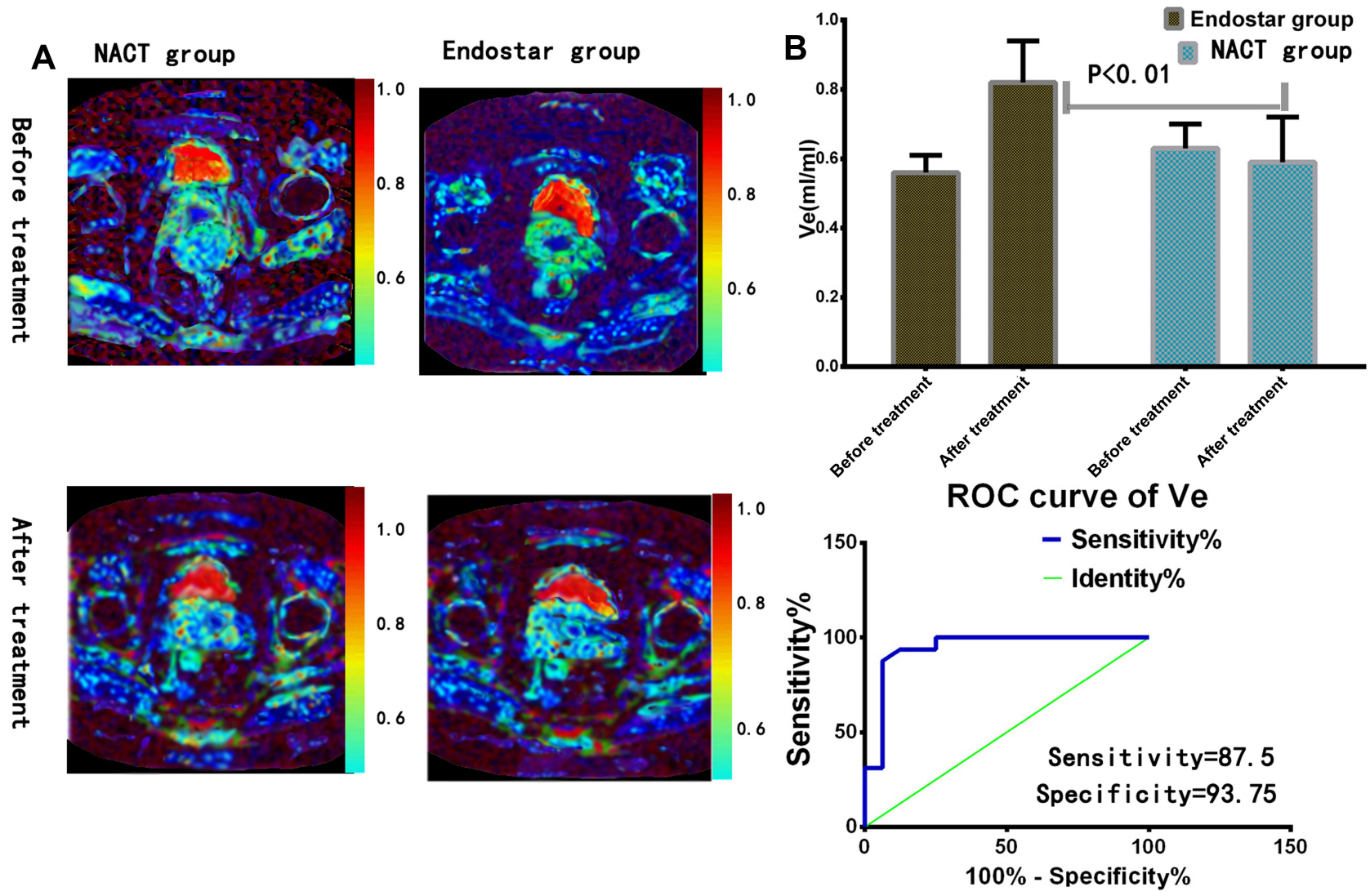

Figure 5 The Ve value detection in endostar treatment. (A) Parametric maps of extravascular extracellular volume fraction (Ve) images were exhibited by DCE-MRI. (B) One week after treatment, the Ve value enhanced obviously in endostar group. ROC analysis between endostar and NACT group was performed. The area under curve (AUC) was $0.9434(\mathrm{P}<0.01)$.

\section{VEGF-Notch Signal Pathway Detection}

The genetic expression of VEGF-Notch signal pathway was analyzed by real-time PCR. The elements of these including VEGFR, Notch1, Notch4, Dll4, Jag1 and hes-1 were obviously downregulated in endostar group except VEGF, comparing to NACT group. This was also confirmed from the protein expression of VEGFR, Jag1, Dll4, Notch1, 4 and hes-1 by Western blot analysis, this proved endostar blocked VEGF-Notch connection via interdicting VEGFR $(\mathrm{P}<0.01)$, see Figure 7.

\section{Discussion}

\section{Current Situation and Problems of Treatment of Cervical Cancer}

Cervical cancer is the most common malignant tumor of the female reproductive tract, and the incidence of cervical cancer is the second in female malignant tumors. About 500,000 new cases of cervical cancer occur worldwide every year, and more than 260,000 women die of cervical cancer every year. The number of new cases in China reached 131,500 per year. Cervical cancer accounts for

Table 5 The Histopathologic Features in Two Groups

\begin{tabular}{|l|l|l|l|l|}
\hline $\mathbf{n I = n 2 = 6 9}$ & Endostar Group & NACT Group & Fisher's Exact Test & P value \\
\hline Deep stromal invasion & $23(33.3 \%)$ & $27(39.1 \%)$ & $\alpha>0.05$ & 0.5954 \\
Excision margin status & $69(100 \%)$ & $69(100 \%)$ & $\alpha>0.05$ & 1.000 \\
Vaginal invasion & $6(8.7 \%)$ & $4(5.7 \%)$ & $\alpha>0.05$ & 0.7445 \\
Parametrial involvement & $3(6.3 \%)$ & $7(10.4 \%)$ & $\alpha>0.05$ & 0.3252 \\
Lymphovascular space involvement. & $21(30.4 \%)$ & $32(46.2 \%)$ & $\alpha<0.05$ & 0.0286 \\
Lymph node metastasis & $6(8.7 \%)$ & $14(20.3 \%)$ & $\alpha<0.05$ & 0.0432 \\
Tumor regression grade $\geq 2 / 3$ & $26(37.7 \%)$ & $14(20.3 \%)$ & $\alpha<0.05$ & 0.0078 \\
\hline
\end{tabular}



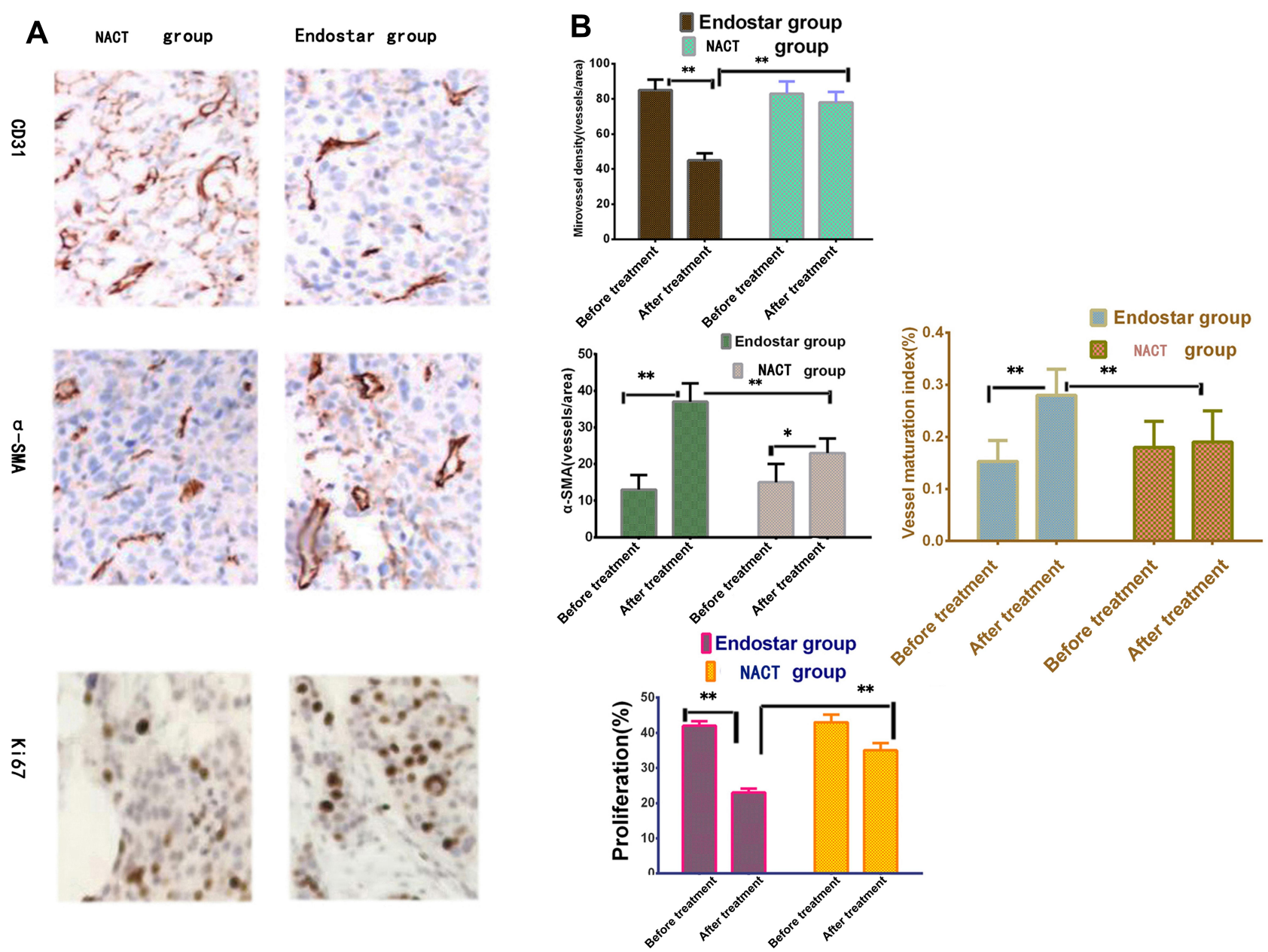

Figure 6 The tumor pathologic detection in endostar treatment. (A) Microvessel density (CD3I staining) relatively reduced in endostar than NACT group (P<0.00I). $\alpha$ SMA staining (pericyte coverage) increased in endostar than NACT group $(\mathrm{P}<0.00 \mathrm{I})$. (B) The ratio of $\alpha$-SMA to CD3I labeling (vessel maturation index, VMI) increased in endostar than NACT group ( $\mathrm{P}<0.00 \mathrm{I}$ ) (Figure above). Tumor proliferating ratio (Ki67 staining) decreased significantly than NACT group ( $\mathrm{P}<0.00 \mathrm{I})$ (Figure below). (Unpaired $t$-test, $* \mathrm{P}<0.05 ; * * \mathrm{P}<0.01)$.

about 53,000 deaths per year, or $18.4 \%$ of all deaths from malignant tumors in women. The incidence of cervical cancer has declined with the increasing use of HPV vaccines, but cervical cancer remains a difficult problem globally. ${ }^{1,5}$

It can be seen that cervical cancer is an important disease that endangers the health and life of Chinese women. Radiation therapy is regarded as a standard treatment just like surgery, but radiation can induce radiation colitis, loss of ovarian function and sexual capacity, Patients with early or locally advanced cervical cancer can be cured by radical surgery, chemoradiotherapy, but cervical cancer often occurs local invasion and lymph node metastasis, leading to treatment failure. Cervical cancer is not sensitive to conventional chemotherapeutic agents, resistance to chemotherapeutic agents limit their treatment effects, so it is necessary to seek new treatment methods. Currently, endostar is used clinically to treat advanced relapsed and metastatic cervical cancer. The platinum-based concurrent chemoradiotherapy maximizes the OS rate and benefits patients with advanced cervical cancer, but the concurrent chemoradiotherapy still fails to effectively solve the problem of tumor proliferation and metastasis, with the OS rate hovering around 50\%.

\section{Endostar Normalization of Tumor Vascularities}

Neovascularization is a necessary condition for tumor growth. Because of the disordered arrangement of tumor blood vessels, the loss of basal cells and peripheral cells, vascular leakage causes the increase of interstitial pressure and imbalance of blood supply. Anti-angiogenic agents 

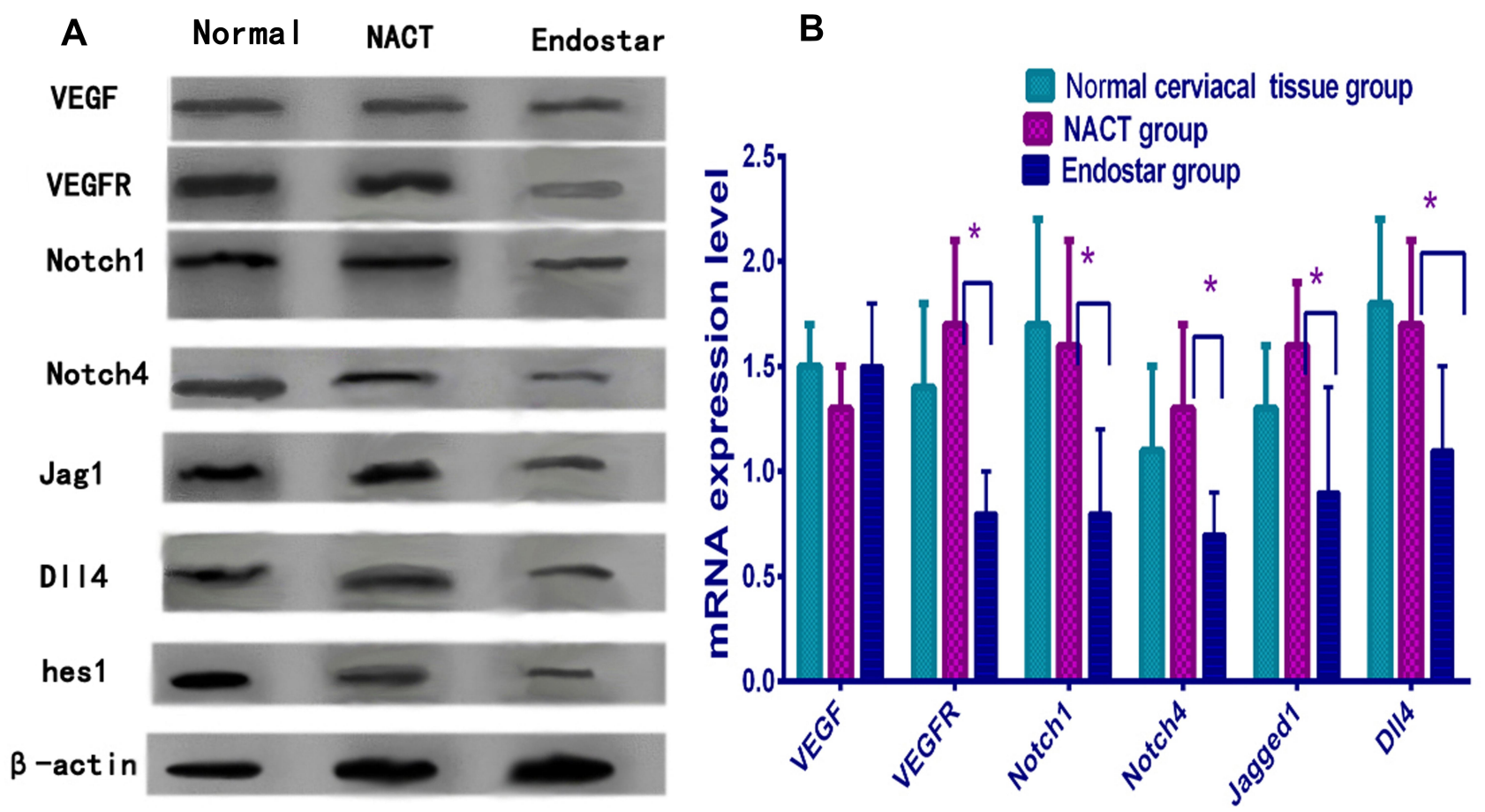

Figure 7 The genetic and protein expression of VEGF-Notch connection. (A) The proteins expression by Western blotting analysis decreased obviously in endostar than NACT group except VEGF. (B) The genes expression by real-time PCR analysis declined also in endostar group than NACT group. These proved endostar blocked VEGF receptors and inhibited VEGF-Notch connection (unpaired $t$-test, ${ }^{*} \mathrm{P}<0.05$ ).

temporarily stabilize abnormal tumor blood vessels by regulating the balance of angiogenic factors, thereby normalizing the irregular vascular structure of tumor, so as to reduce vascular diameter and permeability, improve oxygen delivery and overall vascular responsiveness In this way, cytotoxic chemotherapy agents play a greater role in inhibiting tumor growth. ${ }^{9}$

In recent years, angiogenesis inhibitors combined with standard radiotherapy and platinum-based chemotherapy have become a new focus in the treatment of advanced cervical cancer. In 2005, China independently developed a new type of recombinant human endostatin named endostar. Endostar possesses the function of inhibiting angiogenesis by inhibiting the expression of VEGF and the activity of proteolytic enzyme. Endostatin exerts its antiangiogenesis effect on multiple targets, leading to the dormancy or withdrawal of tumor cells, with clear targeting and no drug resistance. It confirmed endostar could improve chemotherapeutic agent effects in many solid tumors including lung cancer, cervical cancer, etc. ${ }^{10,11}$

On July 20, 2017, endostar-recombinant human endostatin injection was successfully listed on the national medical insurance list, benefiting more patients. Currently, endostar plus chemotherapy has also been used as a first-line treatment for a variety of solid malignancies: cervical cancer, ovarian cancer, non-small cell lung cancer, etc. ${ }^{12,13}$

\section{Endostar Enhances the Therapeutic Efficacy of Chemotherapy Agents}

In this study, we tried to determine whether endostar can enhance effects for cisplatin+paclitaxel (PT) formula in the treatment of local advanced cervical cancer FIGO Staging within IIB2.

In this study, the recent efficacy (2 year) of endostar +NACT was evaluated, it exhibited endostar enhanced chemotherapy response rates in endostar group than NACT group $(89.9 \%$ vs. $72.4 \% \quad \mathrm{P}=0.0155)$ and also increased the disease control rates in endostar group than NACT group ( $95.6 \%$ vs. $76.8 \%, \mathrm{P}=0.0001)$. This results indicated endostar increased chemotherapy effects than NACT alone.

The results exhibited endostar+NACT improved the 2 years progression-free survival (PFS) rates $(97.1 \%$ vs. $84.1 \%, \mathrm{P}=0.0028)$ and increased the LRFS rates in patients with cervical cancer $(95.7 \%$ vs. $85.5 \%$, $\mathrm{P}=0.0329)$ than NACT alone. The results also proved endostar+NACT enhanced the 2-year distance metastasis- 
free survival rates in endostar group $(93.3 \%$ vs. $81.4 \%$, $\mathrm{P}=0.019)$ than NACT group.

In this study, the effects of endostar+NACT in cervical cancer treatment were followed up for 5 yeas, it showed endostar+NACT enhanced longtime (5-year) OS rates $(87.0 \%$ vs. $71.0 \%, \mathrm{P}=0.0087)$ in endostar group than NACT alone.

This indicates endostar+NACT could obtain more benefit for cervical cancer patients, increase ratio of OS and PFS. The rates of local relapse and distance metastasis in endostar group declined than that of NACT group. Endostar administrating could enhance NACT response in treatment for locally advanced cervical cancer, decrease tumor regression and lymphovascular invasion rates. It was reported endostar had the advantage of potential anti-tumor metastasis, Our patients prognosis and pathologic results were consistent with the previous reports in other cancer. ${ }^{14}$

In this study, the adverse reaction of endostar and NACT was closely followed up according to NCI-CTC stand. The results showed that endostar was with mild toxicity and side effects, what more, the adverse responses of NACT such as myelosuppression, gastrointestinal reactions, and weightlessness were relatively reduced than that of NACT group, this indicated endostar relieved NACT adverse effects. The benefit effects may be induced by endostar increasing peripheral tissue vascular perfusion and reducing interstitial pressure.

Endostar has the following advantages, it ACTS directly on vascular endothelial cells, while the proliferation of anticancer drugs is affected by tissue necrosis, fibrosis, and intracellular hypertension, and the effective concentration in tissues cannot reached. The vascular endothelial cell type gene is stable, which is not easy to produce drug resistance, and the tumor cell gene is unstable, which is easy to produce drug resistance. The vascular endothelial cells of primary tumor and metastatic tumor are the same, while the biological differences between primary and metastatic tumors are more obvious, and the response to chemotherapy also varies. The growth rate of tumor endothelial cells is many times faster than that of normal tissue.

\section{The Efficacy of Endostar Determined by DCE-MRI}

In the above study, cervical cancer patients receive NACT therapy to reduce tumor burden to a size fit for surgical operation. The change of tumor volume was monitored by MRI, It exhibited that tumor volumes and maximum diameters further declined in endostar group than NACT group $(\mathrm{P}<0.0001)$, this indicated endostar enhanced NACT tumor volume reduction effects.

DCE-MRI could administrate cervical cancer responsive to NACT treatment, acquire cervical cancer perfusion, permeability condition, quantitively determine contrast iohexol transferred from tumor blood vessel into tissue stroma, the effects of endostar+NACT was evaluated through the imaging. ${ }^{15,16}$

We adopted the endostar+PT project to increase cytotoxic effects of chemotherapy and reduced its side effects.

The DCE-MRI indexes are used in this research included Ktrans, Kep and Ve. Ktrans stands for the amount of contrast agent transferred from blood to tissue and blood vessel permeability. Ve refers to the amount of contrast agent every unit volume in tissue stroma. Kep is ratio of Ktrans and Ve, which refer to the contrast agent transfer from the tissue space to the blood.

The ROC analysis for DCE-MRI parameters were carried out. The ROC analysis showed area under curve values of Ktrans, Ve increased obviously in endostar group after treatment, there was significant different before and after treatment for Ktrans and Ve $(\mathrm{P}<0.0001)$. In contrast, there were no significant differences between before and after treatment in the NACT group ( $\mathrm{p}>0.05)$.

The increase of Ktrans value proved tumor vascular perfusion enhancing, this phenomenon is also confirmed by pathological staining, which exhibited tumor vascular maturation index enhanced at the same time. In the above study, the increase of Ktrans value indicates endostar enhances blood vessel transfer chemotherapeutic agents into tumor tissues so as to enhance cytotoxic agents effects.

The Ve value stands for the out of cellular and vascular space, the higher Ve value, the more amount of contrast or chemotherapeutic agents can be accumulated into the extracellular extravascular space, this was confirmed by our study. The values of Ve increased more obviously in endostar group than NACT group. Both phenomena can be attributed to damages to the tumor cells as a result of cytotoxic and the concurrent anti-angiogenic action.

The kep values were not obviously different in both group before and after treatment. Because Ve and Ktrans values both increased after treatment in endostar group, so the ratio of Ktrans to Ve did not change obviously. Similarly, Ktrans and Ve values in NACT group had little changes before and after treatment, so kep values had little change.

In the study, DCE-MRI as a noninvasive imaging is regarded as a valuable tool for predicting chemotherapy 
treatment response. The ROC analysis was used to determine the optimal cutoff value DCE-MRI parameters. The sensitivity and specificity of Ktrans, Ve and kep were determined by area under curve, DCE-MRI parameters exhibited endostar boosted vascular maturation, so the cervical tumor blood flow increased and interstitial fluid pressure reduced, thereby improving chemotherapeutic agent delivery and effects.

These results exhibited endostar improved tumor perfusion and permeability and increased chemotherapy efficacy and proved DEC-MRI was non-invasive method that could be used to predict for cervical cancer treatment effects and monitor NACT effects in real time. This was consistent with previous reports that DCE-MRI indexes could predict tumor response to treatment and be used as a reliable method to follow up the changes of tumor blood vessel perfusion. ${ }^{17,18}$

\section{The Efficacy of Endostar Determined by Pathology}

The cervical deep stromal invasion invasion, vaginal invasion, parametrial involvement, excision margin status were not obviously different between endostar and NACT group $(\mathrm{P}>0.05)$. However, The tumor regression rates, lymphovascular space involvement, lymph node metastasis were obviously lower in the endostar group than in the NACT group $(\mathrm{P}<0.005)$. The results proved endostar administration could enhance NACT response in treatment for locally advanced cervical cancer and decrease tumor regression rates, lymphovascular metastasis. Two cycles of endostar+TP regiment to cervical cancer patients would acquire a satisfied chemotherapeutic cytotoxic effects with mild adverse events.

\section{Vascular Maturation and Cell Proliferation}

Malignant tumors require angiogenesis to maintain their growth and invasion, and these new vessels are distributed abnormally, with uneven diameters and loose junctions between the endothelial cells. The vascular structures are immature. 9

Before the treatment, CD31 staining rates in endostar group and NACT group was no significantly different between two groups $(\mathrm{P}=0.1362)$. After treatment, CD31 staining rates in endostar group relatively reduced than that of NACT group. There was significant different between two groups $(\mathrm{P}<0.0001)$. $\alpha$-SMA staining were at the same level in two groups before treatment. After treatment, $\alpha$-SMA label in endostar group enhanced dramatically than that NACT group. The change of $\alpha$-SMA to CD31 ratio resulted in a higher VMI in endostar group. $\alpha$ SMA label and VMI were higher in endostar group after endostar treatment $(\mathrm{P}<0.0001)$. This proved endostar made tumor vascular structure more maturely than that of NACT group.

Before the treatment, Ki67 positive staining exhibited the same proliferative level in endostar group and NACT group, there was no significantly different between two groups. After treatment, Ki67 positive staining rates in endostar group were greatly reduced than that of NACT group $(\mathrm{P}<0.0001)$. This proved the tumor proliferative rates in endostar group were obviously inhibited than that of NACT group.

The pathology results indicated when endostar was given, cervical tumor pericyte rates increased, MVD reduced. Vascular basement membrane and endothelial cell structure was tidied up and vascular structural maturation index was increased.

The above study indicated that endostar could make the tumor blood vessels uniform and improve the hemodynamic characteristics, so as to deliver chemotherapeutic agents more effectively. In this way, cytotoxic chemotherapeutic drugs play a greater role in inhibiting tumor growth.

\section{VEGF-Notch Connection Detection}

Notch signaling is an evolutionarily firmly conserved signaling system. It widely exists in all known animal cells and regulates the proliferation, differentiation and apoptosis of tissues and cells. VEGF-notch signal cause tumor abnormal vascular growth through controlling tip and stalk cells balance. ${ }^{19,20}$

The regulating mechanism of VEGF-Notch connection was explored in this study. The results showed the genes and proteins expression of VEGFR2, Notch1, Notch4, Dll4, JAG1 were obviously downregulated in endostar group comparing to NACT group. This proved VEGFNotch connection was blocked by endostar in the endostar group. But the expression changes of tip and stalk cells in this course need further exploration.

To sum up, we adopted endostar+NACT in cervical cancer patients within FIGO staging IIB2, it was proved endostar+NACT could increase ratio of OS and PFS for cervical cancer patients and endostar was with mild adverse reaction than that of NACT along. DEC-MRI as a non-invasive tool was used to evaluate treatment effects 
in real time, the images exhibited endostar improved tumor perfusion and permeability, further enhanced NACT tumor volume reduction efficacy. The pathological detection proved endostar enhanced tumor vascular structural maturation, increased deliver chemotherapeutic agents effectively, cytotoxic agents play a greater role in inhibiting tumor growth. The VEGF-Notch connection blocked by endostar may play a key role this treatment.

The limitation for this study was that it only consisted of cervical cancer patients with FIGO staging IIB2, these patients in the earlier disease stages could exhibit the maximum therapeutic benefit of the anti-angiogenic effects. Whether endostar can benefit for patients with advanced FIGO staging needs further exploration, a multicenter prospective controlled study with cervical cancer all FIGO staging may confirm thiese findings. ${ }^{21}$

\section{Conclusions}

Endostar temporarily restored vascular homeostasis in cervical cancer within FIGO staging IIB2, provided a new way to reverse chemotherapeutic agents resistance in cervical cancer, increased patient survival time. The combination of DEC-MRI and pathology as reliable tools for evaluation the treatment effects for patients.

The anti-angiogenic agent endostar can suspend immature blood vessel formation. Endostar+NACT formula could reverse abnormal tumor vascular structure. Endostar improved tumor vessel maturation, increased chemotherapy effect at the same time. The VEGF-Notch connection blocked by endostar may play a key role this treatment.

\section{Abbreviations}

PT, cisplatin+paclitaxel; NACT, neoadjuvant chemotherapy; KPS, Karnofsky; RECIST, response evaluation criteria in solid tumors; FIGO, international federation of gynecology and obstetrics; CR, complete response; PR, partial response; $\mathrm{SD}$, stable disease; $\mathrm{PD}$, progressive disease; DCR, Disease control rate; OS, overall survival; PFS, progression-free survival; LRFS, local relapse-free survival; DMFS, distant metastasis-free survival; NCI, National Cancer Institute; DEC-MRI, dynamic contrastenhanced magnetic resonance imaging; TE, echo time; TR, repetition time; Ktrans, volume transfer constant; Ve, extravascular extracellular volume fraction; kep, Ktrans/ve, the efflux constant; ROC, receiver operating characteristic curve; VMI, vessel maturation index; VEGF, vascular endothelial growth factor; VEGFR, Vascular Endothelial Growth Factor Receptor; cDNA, complementary deoxyribonucleic acid; RT-PCR, real time polymerase chain reaction; SDS-PAGE, sodium dodecyl sulfate polyacrylamide gel electrophoresis; PVDF, transferred to polyvinylidene difluoride; BSA, bovine serum albumin; ANOVA, Analysis of Variance.

\section{Data Sharing Statement}

All data sets in this study are within the article as well as its figures and tables, there are not other data and materials available.

\section{Ethics Approval and Consent to Participate}

This study was conducted in accordance with the Declaration of Helsinki and approved by the Ethics committee of Zhabei Central Hospital (No. 2015-35). The personal protection committees and scientific committees of Zhabei Central Hospital approved this study at the same time. All patients in this study were thoroughly informed of the benefits, curative effects, potential risks of the condition and management alternatives, treatment costs and uncertainties related to cervical cancer radical operation, or a NACT protocol treatment followed by surgery. All patients gave written consent to participate in the study.

\section{Consent for Publication}

The author consents this article for publication.

\section{Acknowledgments}

The author thanks medical staff from Zhabei central hospital for providing the useful advice and cooperation. The author thanks Professor Yuezhi Zhang and Professor Xiaohan Wang from Chifeng Second Hospital for their help with pathologic technology.

\section{Author Contributions}

The author made substantial contributions to conception and design, acquisition of data, analysis and interpretation of data; took part in drafting the article, revising it critically for important intellectual content; agreed to submit to the current journal; gave final approval of the version to be published; and agree to be accountable for all aspects of the work.

\section{Funding}

The project was funded by Zhabei Central Hospital (No.2015-35). 


\section{Disclosure}

The author reports no conflicts of interest for this work.

\section{References}

1. Finocchario-Kessler S, Wexler C, Maloba M, et al. Cervical cancer prevention and treatment research in Africa: a systematic review from a public health perspective. BMC Womens Health. 2016;16:29.

2. Wang $\mathrm{Y}, \mathrm{Hu} \mathrm{D}, \mathrm{Hu} \mathrm{X}$, et al. Assessing the early response of advanced cervical cancer to neoadjuvant chemotherapy using intravoxel incoherent motion diffusion-weighted magnetic resonance imaging: a pilot study. Chin Med J (Engl). 2016;129(6):665-671. doi:10.41 03/0366-6999.177995

3. Xu Q, Gu J, Lv Y, et al. Angiogenesis for tumor vascular normalization of Endostar on hepatoma 22 tumor-bearing mice is involved in the immune response. Oncol Lett. 2018;15(3):3437-3446.

4. Sun C, Tian X, Liu Z, et al. Radiomic analysis for pretreatment prediction of response to neoadjuvant chemotherapy in locally advanced cervical cancer: a multicentre study. EBio Medicine. 2019;46:160-169.

5. Bhatla N, Aoki D, Sharma DN, Sankaranarayanan R. FIGO cancer report 2018: cancer of the cervix uteri. Int $J$ Gynecol Obstet. 2018;143(Suppl 2):22-36. doi:10.1002/ijgo.12611

6. Schulz KF, Altman DG, Moher D, for the CONSORT Group (2010) CONSORT 2010 Statement: Updated Guidelines for Reporting Parallel Group Randomised Trials. PLoS Med 7(3): e1000251. doi. org/10.1371/journal.pmed.1000251

7. Chen X, Chen G, Guo-hui X, et al. Tumor size at magnetic resonance imaging association with lymph node metastasis and lymphovascular space invasion in resectable cervical cancer: a multicenter evaluation of surgical specimens. Int $J$ Gynecol Cancer. 2018;28(8):1545-1552.

8. Dworak O, Keilholz L, Hoffmann A. Pathological features of rectal cancer after preoperative radiochemotherapy. Int $J$ Colorectal Dis. 1997;12:19-23. doi:10.1007/s003840050072

9. Sorace AG, Chad Quarles C, Whisenant JG, et al. Trastuzumab improves tumor perfusion and vascular delivery of cytotoxic therapy in a murine model of HER2+ breast cancer: preliminary results. Breast Cancer Res Treat. 2016;155(2):273-284. doi:10.1007/ s10549-016-3680-8

10. Li W, Quan Y, Li Y, et al. Monitoring of tumor vascular normalization: the key points from basic research to clinical application. Cancer Manag Res. 2018;10:4163-4172. doi:10.2147/CMAR. $\mathrm{S} 174712$
11. Guan Y, A L, Xiao W, et al. The efficacy and safety of Endostar combined with chemoradiotherapy for patients with advanced, locally recurrent nasopharyngeal carcinoma. Oncotarget. 2015;6(32):33 926-33934. doi:10.18632/oncotarget.5271

12. Wang Y, Nie J, Dai L, et al. Efficacy and toxicities of gemcitabine and cisplatin combined with endostar in advanced thymoma and thymic carcinoma. Thorac Cancer. 2019;10(1):17-23. doi:10.1111/ 1759-7714.12891

13. Li Y, Huang P, Peng H, et al. Antitumor effects of Endostar(rh-endostatin) combined with gemcitabine in different administration sequences to treat Lewis lung carcinoma. Cancer Manag Res. 2019;11:3469-3479. doi:10.2147/CMAR.S192868

14. Zhao X, Pan J, Song Y, et al. Recombined human endostatin (Endostar) enhances cisplatin delivery and potentiates hemotherapy by decompressing colorectal cancer vessels. Int J Clin Exp Pathol. 2017;10(11):10792-10801.

15. Pan J, Zhu S, Huang J, et al. Monitoring the process of endostar-induced tumor vascular normalization by non-contrast intravoxel incoherent motion diffusion-weighted. MRI Front Oncol. 2018;8:524. doi:10.3389/fonc.2018.00524

16. Tao X, Wang L, Hui Z, et al. DCE-MRI perfusion and permeability parameters as predictors of tumor response to CCRT in patients with locally advanced NSCLC. Sci Rep. 2016;6:35569. doi:10.1038/ srep35569

17. Qiao P, Zhao P, Gao Y, et al. Differential study of DCE-MRI parameters in spinal metastatic tumors, brucellar spondylitis and spinal tuberculosis. Chin J Cancer Res. 2018;30(4):425-431. doi:10.21147/ j.issn.1000-9604.2018.04.05

18. Zhu L, Zhu L, Shi H, et al. Evaluating early response of cervical cancer under concurrent chemo-radiotherapy by intravoxel incoherent motion MR imaging. BMC Cancer. 2016;16:79. doi:10.1186/s12885016-2116-5

19. Chen Y, Zhao B, Zhu Y, Zhao H, Ma C. HIF-1-VEGF-Notch mediates angiogenesis in temporomandibular joint osteoarthritis. Am J Transl Res. 2019;11(5):2969-2982.

20. Colombo M, Mirandola L, Chiriva-Internati M, et al. Cancer cells exploit notch signaling to redefine a supportive cytokine milieu. Front Immunol. 2018;9:1823.

21. Caporarello N, Lupo G, Olivieri M, et al. Classical VEGF, Notch and Ang signalling in cancer angiogenesis, alternative approaches and future directions. Mol Med Rep. 2017;16(4):4393-4402. doi:10.38 92/mmr.2017.7179
OncoTargets and Therapy

\section{Publish your work in this journal}

OncoTargets and Therapy is an international, peer-reviewed, open access journal focusing on the pathological basis of all cancers, potential targets for therapy and treatment protocols employed to improve the management of cancer patients. The journal also focuses on the impact of management programs and new therapeutic
Dovepress

agents and protocols on patient perspectives such as quality of life, adherence and satisfaction. The manuscript management system is completely online and includes a very quick and fair peer-review system, which is all easy to use. Visit http://www.dovepress.com/ testimonials.php to read real quotes from published authors. 